\title{
Instrumento de evaluación para el desarrollo en edades tempranas de Frugone \& Miño
}

\author{
Assessment instrument for early development of Frugone \& Miño \\ *Sebastián Díaz, ${ }^{* *}$ Bastián Parada, ${ }^{* * *}$ Katherine Rojas, ${ }^{* * *}$ Diego Frugone, \\ **** Magdalena Miño, ${ }^{* * * *}$ Cesar Faúndez
}

Díaz, S., Parada, B., Rojas, K., Frugone, D., Miño, M., \& Faúndez, C. (2019). Instrumento de evaluación para el desarrollo en edades tempranas de Frugone \& Miño. Revista Ciencias de la Actividad Física UCM, N²0(2), julio-diciembre, 1-10. DOI: http://doi.org/10.29035/rcaf.20.2.8

\section{RESUMEN}

Objetivo: Proponer una batería para evaluar el desarrollo motor para estudiantes de primer ciclo básico. Metodología: se evaluó una muestra de 190 estudiantes, pertenecientes a tres establecimientos educacionales de la ciudad de Talca, Chile. El rango de edad oscila entre los 5 y 9 años de edad. Resultados: El instrumento evidenció valores aceptables confiabilidad y consistencia interna (Alpha de Cronbach 0,829) Los puntos de corte adoptados son: p<33 Inicial, zp33 a p66 Elemental y zp66 maduro. Los resultados demuestran que no existen diferencias entre ambos géneros en relación a las habilidades motrices básicas de locomoción, manipulación y equilibrio. Conclusión: test de Frugone \& Miño es un instrumento fiable para contribuir de forma segura y confiada en la evaluación de los niveles del desarrollo motor en estudiantes escolarizados de primer ciclo básico, lo que sugiere su uso y aplicación en el colegio para monitorizar el progreso del desempeño motor de los alumnos de ambos sexos.

\section{PALABRAS CLAVE}

Desarrollo motor, niños, validación, locomoción, manipulación, equilibrio.

\begin{abstract}
Objective: Propose a cognitive assessment (battery) to evaluate the motor development of students during the first basic cycle. Methodology: A sample of 190 students was evaluated, belonging to three educational establishments in the city of Talca, Chile. The age range varied between 5 and 9 years of age. Results: The instrument showed acceptable values, reliability and internal consistency (Cronbach's Alpha 0.829). The cutting points adopted are: p <33 Initial, $\geq$ p33 to p66 Elemental and zp66 mature. Conclusion: Frugone \& Miño test is a reliable instrument to safely and confidently contribute to the evaluation of the motor development levels in students enrolled in the first basic cycle, which suggests its use and application in school to monitor the progress of the motor performance of students from both sexes. The results show that there are no differences between both genders in relation to the basic motor skills of locomotion, manipulation and balance.
\end{abstract}

\section{Key words}

Motor development, Validation, Locomotion, Manipulation, Balance.

\footnotetext{
* Gimnasio Energy, Linares, Chile. ** Escuela Punta de Diamante, San Clemente, Chile. ${ }^{* * *}$ Licenciada en educación, Universidad Católica del Maule. Talca, Chile ${ }^{* * * *}$ Colegio Santo Tomás de Talca, Talca, Chile. ${ }^{* * * * *}$ Depto. Ciencias de la Actividad Física. Facultad de Ciencias de la Educación. Universidad Católica del Maule, Talca, Chile.
} 
Díaz, S., Parada, B., Rojas, K., Frugone, D., Miño, M., \& Faúndez, C. (2019). Instrumento de evaluación para el desarrollo en edades tempranas de Frugone \& Miño. Revista Ciencias de la Actividad Física UCM, №20(2), julio-diciembre, 1-10. DOI: http://doi.org/10.29035/rcaf.20.2.8

\section{INTRODUCCIÓN}

El desarrollo motor es un proceso que se lleva a cabo mediante el progreso de movimientos simples y desorganizados para alcanzar otras habilidades más complejas, considerando al movimiento, parte fundamental del desarrollo integral de los niños (Gallahue, \& Ozmun, 2006).

Además, Doussoulin (2003) afirma que "el desarrollo motor, es un proceso multidimensional que incluye cambios en el plano motor, intelectual, emocional, social y sensorial". La primera etapa escolar es realmente trascendental, debido a que es en este período donde los estudiantes alcanzan el dominio de una serie de habilidades motrices que van a contribuir en su maduración integral, incorporando tanto la dimensión intelectual como afectiva. Asimismo, se debe tener en cuenta que, cada niño posee su propia secuencia de desarrollo, la cual está en directa relación con su maduración, por lo cual puede variar en características y calidad entre un niño u otro (Doussoulin, 2003).

Desde que en 1948 la Organización Mundial de la Salud definió la «salud» como el completo estado de bienestar físico, mental y social, se han desarrollado numerosas investigaciones que están permitiendo traducir esta definición conceptual en métodos u objetivos que, mediante validaciones o instrumentos diversos, generan escalas e índices para facilitar la medición de las dimensiones que conforman el estado de salud y bienestar.

De hecho, el tema del desarrollo motor ha adquirido gran interés en las últimas décadas (Le Boulch, 1995, 1997, Da Fonseca, 1996, Escriba, 1999, Ulrich, 2000, Pascucci et al. 2004, Fritz \& Vargas, 2013), ya que los niños, por su naturaleza, requieren y necesitan moverse libremente. Pero el desarrollo de la sociedad actual y lo artificial de la vida moderna, cada día les presenta mayores obstáculos; falta de espacios adecuados, menos áreas verdes, menos tiempo con sus padres, exceso de actividades sedentarias (televisión, computadores) y jornadas escolares extensas, lo que ha contribuido a la reducción y disminución de instancias de movimiento, y con esto, al menor desarrollo motor durante la infancia. En esta línea, la escuela y los profesores de educación física juegan un rol fundamental en el proceso de evaluación continua y monitoreo de las conductas motrices de los niños por lo cual se vuelve fundamental contar con test para evaluar este aspecto desde la escuela .

Balestrini (1997) plantea: "Una vez que se han definido y diseñado los instrumentos y procedimientos de recolección de datos, atendiendo al tipo de estudio de que se trate, antes de aplicarlos de manera definitiva en la muestra seleccionada, es conveniente someterlos a prueba, con el propósito de establecer la validez de éstos, en relación con el problema investigado". Además, agrega que "toda investigación, en la medida que sea posible, debe permitir ser sometida a ciertos correctivos a fin de refinarlos y validarlos". En esta línea, la validación de un método es el proceso para confirmar que el procedimiento utilizado, para una prueba en concreto, es adecuado para el uso previsto. Los resultados de la validación del método pueden utilizarse para juzgar la calidad, la fiabilidad y la constancia de los resultados.

La confiabilidad es definida como la precisión con que el test mide lo que mide en una población determinada y en condiciones normales de aplicación (Aiken, 1996; Anastasi, 1986). En consecuencia, dentro de esta categoría el Alfa de Cronbach es, sin duda, el más ampliamente utilizado por los investigadores (Ledesma, Molina, Valero, 2002), especialmente cuando se trata de analizar la consistencia interna de instrumentos, sobre todo cuando se tiene una única medición en el tiempo, con lo que es posible ahorrar tiempo (Fuentes-Lopez, Puño, De arruda, Cossio-Bolaños, 2014). 


\section{OBJETIVO}

Proponer una batería para evaluar el desarrollo motor para estudiantes de primer ciclo básico, en escolares de la ciudad de Talca, con el fin de definir en qué estado clasifican, tanto en un estado inicial, elemental o maduro.

\section{METODOS}

\section{Tipo de estudio y muestra}

El presente estudio es de tipo descriptivo de corte transversal. Se evaluó una muestra de 190 estudiantes, pertenecientes a tres establecimientos educacionales de la ciudad de Talca, Chile. El rango de edad oscila entre los 5 y 9 años de edad. La selección de la muestra fue no-probabilística. Los alumnos fueron reclutados de forma accidental entre los que cursaban de primero a cuarto básico. Se solicitó a todos los alumnos la firma de consentimiento informado de sus padres, guiado por los resguardos éticos de la declaración de Helsinky expuestos en los documentos del Comité de Ética de la Universidad Católica del Maule (87/2017). Todos los escolares al momento de la aplicación del instrumento realizaban por lo general como parte de las sesiones de Educación Física 90 minutos de contenidos de actividades físicas, los que según el sistema educativo chileno deben ser realizados dos veces por semana.

\section{Procedimientos para recolección de da- tos:}

El Test de Frugone \& Miño es un test de habilidades motrices básicas que consiste en la evaluación de las habilidades de locomoción, manipulación y equilibrio por medio de 12 pruebas motrices, el cual puede ser aplicado en niños de 5 a 10 años.

Para demostrar la validez del constructo, con base en la teoría o esquema teórico elaborado a partir de la operacionalización de la variable, se utilizó el coeficiente de Cronbach (1984), basado en la varianza de los ítems del instrumento. Se calcularon puntos de corte adoptados: $p<33$ Inicial, zp33 a p66 Elemental y zp66 maduro.

\section{Análisis estadísticos}

La distribución normal de la muestra, en cada una de las variables de análisis, fue verificada a través de la prueba de normalidad Kolmogórov-Smirnov. La base de datos fue almacenada y procesada en planillas Excel y analizada a través del programa SPSS 18.0. Los datos se analizaron por medio de estadística descriptiva de frecuencias (n), porcentajes (\%), media aritmética (X), desviación estándar (DE), mínimo y máximo (Min y Máx). Para verificar la fiabilidad y confiabilidad (consistencia interna) del instrumento, se aplicó el coeficiente de Alpha de cronbach.

\section{RESULTADOS}

La Tabla 1, muestra las características generales de la muestra estudiada, donde se observa el promedio de edad de los alumnos estudiados (5 a 9 años) para cada nivel. Los alumnos, en el momento en que fueron evaluados, realizaban clases de educación física dos veces por semana durante 90 min 45 minutos, la hora pedagógica dentro del proceso de enseñanza-aprendizaje, lo que corresponde a un total de 180 minutos por semana. 
Díaz, S., Parada, B., Rojas, K., Frugone, D., Miño, M., \& Faúndez, C. (2019). Instrumento de evaluación para el desarrollo en edades tempranas de Frugone \& Miño. Revista Ciencias de la Actividad Física UCM, N²0(2), julio-diciembre, 1-10. DOI: http://doi.org/10.29035/rcaf.20.2.8

Tabla 1

Características de la muestra estudiada.

\begin{tabular}{lccccccccccc}
\hline & \multicolumn{4}{c}{} & \multicolumn{2}{c}{ Edad } & \multicolumn{2}{c}{ Locomoción } & \multicolumn{2}{c}{ Manipulación } & \multicolumn{2}{c}{ Equilibrio } & \multicolumn{2}{c}{ Total } \\
\hline Nivel & $\mathbf{n}$ & $\mathrm{X}$ & $\mathrm{DE}$ & $\mathrm{X}$ & $\mathrm{DE}$ & $\mathrm{X}$ & $\mathrm{DE}$ & $\times$ & $\mathrm{DE}$ & $\times$ & $\mathrm{DE}$ \\
\hline Primero & 69 & 5,81 & 0,43 & 43,01 & 3,49 & 28,88 & 3,39 & 45,17 & 2,92 & 117,07 & 7,24 \\
\hline Segundo & 41 & 7,02 & 0,27 & 40,10 & 3,07 & 27,20 & 3,46 & 44,17 & 6,24 & 111,46 & 9,65 \\
\hline Tercero & 36 & 7,86 & 0,35 & 43,28 & 3,35 & 30,25 & 2,50 & 46,03 & 2,81 & 119,55 & 46,16 \\
\hline Cuarto & 44 & 8,89 & 3,27 & 46,16 & 2,45 & 30,48 & 2,06 & 45,77 & 2,62 & 122,40 & 5,34 \\
\hline
\end{tabular}

Los resultados mostrados en la Tabla 2 permiten destacar los valores obtenidos para confiabilidad de la batería de desarrollo motor propuesta por Frugone \& Miño, por medio de la consistencia DE interna, para lo cual se utilizó el coeficiente de alfa Cronbach, encontrándose fiabilidad entre los ítems evaluados.

Los valores obtenidos de alfa Cronbach oscilaron entre: para Locomoción entre 0,657, para Manipulación 0,701, y para Equilibrio 0,828. Por lo tanto, destacamos que los valores de confiabilidad, tanto de forma individual para cada sub ítem y de forma total del instrumento $(0,829)$, muestran altos niveles de confiabilidad.

Tabla 2

Valores del coeficiente de Cronbach (alfa) para cada dimensión del instrumento.

\begin{tabular}{lc}
\hline Dimensiones & Alfa de Cronbach \\
\hline Locomoción & 0,657 \\
\hline Manipulación & 0,821 \\
\hline Equilibrio & 0,822 \\
\hline Total batería & 0,829 \\
\hline
\end{tabular}

En la Tabla 3, se observa la distribución de percentiles. A partir de esta tabla se puede evaluar a los niños. Los que están por debajo del percentil 33 presentan un nivel inicial en lo que respecta a su desarrollo motor; los que están entre el p33 al p66, presentan un nivel elemental y los que están arriba del p66 tienen maduro de motricidad. Esta clasificación se llevó a cabo tanto para el total del instrumento como para cada una de sus dimensiones.

\section{Tabla 3}

Distribución de percentiles para calcular las categorías para cada una de las dimensiones de desarrollo motor.

\begin{tabular}{lcccc}
\hline & N & Inicial & Elemental & Maduro \\
\hline Locomoción & 190 & $<41$ & $41-45$ & $>45$ \\
\hline Manipulación & 190 & $<29$ & $29-31$ & $>31$ \\
\hline Equilibrio & 190 & $<45$ & $45-47$ & $>47$ \\
\hline Total & 190 & $<116$ & $116-122$ & $>122$ \\
\hline
\end{tabular}

Según la Tabla 4, en cuanto a la habilidad motriz de locomoción, los estudiantes de segundo básico presentan el mayor porcentaje en cuanto al estado de desarrollo motor inicial con un 65,9\%, mientras que los estudiantes de cuarto básico representan el menor porcentaje con tan solo 4,5\%. En relación al estado de desarrollo motor elemental, los alumnos de primero básico tienen el porcentaje más alto con un 36,2\% respecto a los estudiantes de cuarto básico, que nuevamente presentan el porcentaje más bajo respecto a este estado con tan solo $25 \%$. Finalmente, en cuanto al estado de desarrollo motor maduro, los estudiantes de cuarto básico son los que lideran la estadística, debido a que representan el 70,5\% en comparación con los estudiantes de segundo 
básico donde ninguno forma parte de este estado de desarrollo motor en la habilidad motriz de locomoción. Por otra parte, en la habilidad motriz de manipulación, los estudiantes de segundo básico representan el mayor porcentaje respecto al estado de desarrollo motor inicial con un 68,3\% en comparación con los alumnos de tercero básico que obtuvieron el menor porcentaje con un 19,4\%; en cuanto al estado de desarrollo motor elemental, los estudiantes de tercero básico alcanzaron el mayor porcentaje con un $38,9 \%$ en contraste con los alumnos de segundo básico, que obtuvieron el menor porcentaje con un 26,8\%; por último, en cuanto al estado de desarrollo motor maduro, los estudiantes de cuarto básico representan un $47,7 \%$, muy por encima de los estudiante de segundo, que solo obtuvieron un 4,9\% en este ítem.

Finalmente, en la habilidad motriz de equilibrio, los estudiantes de segundo básico representan el mayor porcentaje en el estado de desarrollo motor inicial, respecto a los alumnos de tercero básico que poseen el menor porcentaje con 25\%; en el estado de desarrollo motor elemental, los estudiantes de primero básico alcanzaron el mayor porcentaje con un 40,6\%, mientras que los estudiantes de segundo básico obtuvieron el menor porcentaje con 22\%; por último, en el estado de desarrollo motor maduro, los estudiantes de tercero básico alcanzaron el mayor porcentaje con un 38,9\%.

Tabla 4

Valores porcentuales y clasificación por indicadory dimensión del instrumento de desarrollo motor de los alumnos evaluados.

\begin{tabular}{|c|c|c|c|c|c|c|}
\hline & Estado & Primero & Segundo & Tercero & Cuarto & Total \\
\hline \multirow{3}{*}{ Locomoción } & Inicial & $34,8 \%$ & $65,9 \%$ & $33,3 \%$ & $4,5 \%$ & $34,2 \%$ \\
\hline & Elemental & $36,2 \%$ & $34,1 \%$ & $38,9 \%$ & $25,0 \%$ & $33,7 \%$ \\
\hline & Maduro & $29,0 \%$ & $0,0 \%$ & $27,8 \%$ & $70,5 \%$ & $32,1 \%$ \\
\hline \multirow{3}{*}{ Manipulación } & Inicial & $46,4 \%$ & $68,3 \%$ & $19,4 \%$ & $25,0 \%$ & $41,1 \%$ \\
\hline & Elemental & $31,9 \%$ & $26,8 \%$ & $38,9 \%$ & $27,3 \%$ & $31,1 \%$ \\
\hline & Maduro & $21,7 \%$ & $4,9 \%$ & $41,7 \%$ & $47,7 \%$ & $27,9 \%$ \\
\hline \multirow{3}{*}{ Equilibrio } & Inicial & $39,1 \%$ & $46,3 \%$ & $25,0 \%$ & $36,4 \%$ & $37,4 \%$ \\
\hline & Elemental & $42,0 \%$ & $24,4 \%$ & $30,6 \%$ & $36,4 \%$ & $34,7 \%$ \\
\hline & Maduro & $18,8 \%$ & $29,3 \%$ & $44,4 \%$ & $27,3 \%$ & $27,9 \%$ \\
\hline \multirow{3}{*}{ Total } & Inicial & $36,2 \%$ & $70,7 \%$ & $27,8 \%$ & $13,6 \%$ & $36,8 \%$ \\
\hline & Elemental & $40,6 \%$ & $22,0 \%$ & $33,3 \%$ & $25,0 \%$ & $31,6 \%$ \\
\hline & Maduro & $23,2 \%$ & $7,3 \%$ & $38,9 \%$ & $61,4 \%$ & $31,6 \%$ \\
\hline
\end{tabular}

En relación con los 69 estudiantes de primer nivel básico, se observa que el puntaje promedio máximo se logró en la habilidad motriz de equilibrio; por otra parte, se aprecia que, si bien no existe una gran diferencia en el puntaje promedio de las tres habilidades motrices básicas entre damas y varones, hay una leve superioridad de estos últimos. Tabla 5. 
Díaz, S., Parada, B., Rojas, K., Frugone, D., Miño, M., \& Faúndez, C. (2019). Instrumento de evaluación para el desarrollo en edades tempranas de Frugone \& Miño. Revista Ciencias de la Actividad Física UCM, № 20(2), julio-diciembre, 1-10. DOI: http://doi.org/10.29035/rcaf.20.2.8

Tabla 5

Valores medios, desviación estándar por genero del instrumento de desarrollo motor de los alumnos evaluados de primero básico.

\begin{tabular}{lcccccccccc}
\hline & \multicolumn{3}{c}{ Total } & \multicolumn{4}{c}{ Damas } & \multicolumn{5}{c}{ Varones } & p-valor \\
\cline { 2 - 13 } & $\mathrm{N}$ & $\times$ & $\mathrm{DE}$ & $\mathrm{N}$ & $\times$ & $\mathrm{DE}$ & $\mathrm{N}$ & $\times$ & $\mathrm{DE}$ & \\
\hline Locomoción & 69 & 43,01 & 3,49 & 37 & 42,97 & 3,670 & 32 & 43,06 & 3,33 &, 916 \\
\hline Manipulación & 69 & 28,88 & 3,39 & 37 & 28,84 & 3,379 & 32 & 28,94 & 3,46 &, 904 \\
\hline Equilibrio & 69 & 45,17 & 2,92 & 37 & 44,95 & 3,091 & 32 & 45,44 & 2,73 &, 490 \\
\hline Total & 69 & 117,07 & 7,24 & 37 & 116,76 & 7,91 & 32 & 117,44 & 6,49 &, 700 \\
\hline
\end{tabular}

En segundo nivel básico, de los 41 estudiantes evaluados, 18 fueron damas y 23 varones, alcanzando el puntaje promedio máximo en la habilidad motriz de equilibrio, al igual que los estudiantes de primer nivel básico; se puede apreciar una tendencia, donde los varones obtuvieron mejores re- sultados que las damas en las actividades relacionadas con las habilidades motrices básicas de locomoción y manipulación, sin embargo, las damas presentan mejores resultados que los varones en las actividades relacionadas a la habilidad motriz de equilibrio. Tabla 6.

Tabla 6

Valores medios, desviación estándar por genero del instrumento de desarrollo motor de los alumnos evaluados de segundo básico.

\begin{tabular}{lcccccccccc}
\hline & \multicolumn{3}{c}{ Total } & \multicolumn{4}{c}{ Damas } & \multicolumn{5}{c}{ Varones } & p-valor \\
\cline { 2 - 13 } & $N$ & $X$ & DE & $N$ & $X$ & DE & $N$ & $X$ & DE & \\
\hline Locomoción & 41 & 40,10 & 3,072 & 18 & 39,94 & 2,461 & 23 & 40,22 & 3,529 & 0,782 \\
\hline Manipulación & 41 & 27,20 & 3,466 & 18 & 26,56 & 2,833 & 23 & 27,70 & 3,878 & 0,302 \\
\hline Equilibrio & 41 & 44,17 & 6,241 & 18 & 45,44 & 2,526 & 23 & 43,17 & 7,970 & 0,253 \\
\hline Total & 41 & 111,46 & 9,65 & 18 & 111,94 & 4,90 & 23 & 111,09 & 12,27 & 0,782 \\
\hline
\end{tabular}

En los 36 estudiantes de tercer nivel básico se observa que el puntaje promedio máximo se alcanzó en la habilidad motriz de equilibrio, en relación a la comparación entre damas y varones; estos últimos solo lograron mejores resultados que las damas en las actividades relacionadas a la habilidad motriz de locomoción, ya que tanto en las actividades relacionadas con la manipulación, así como también en equilibrio, fueron las damas quienes obtuvieron una pequeña superioridad. Tabla 7. 
Tabla 7

Valores medios, desviación estándar por genero del instrumento de desarrollo motor de los alumnos evaluados de tercero básico.

\begin{tabular}{lccccccccccc}
\hline & \multicolumn{3}{c}{ Total } & \multicolumn{4}{c}{ Damas } & \multicolumn{5}{c}{ Varones } & p-valor \\
\cline { 2 - 13 } & $\mathrm{N}$ & $\times$ & $\mathrm{DE}$ & $\mathrm{N}$ & $\times$ & $\mathrm{DE}$ & $\mathrm{N}$ & $\times$ & $\mathrm{DE}$ & \\
\hline Locomoción & 36 & 43,28 & 3,35 & 18 & 42,33 & 2,87 & 18 & 44,22 & 3,606 &, 091 \\
\hline Manipulación & 36 & 30,25 & 2,50 & 18 & 30,44 & 2,41 & 18 & 30,06 & 2,645 &, 647 \\
\hline Equilibrio & 36 & 46,03 & 2,81 & 18 & 46,44 & 2,75 & 18 & 45,61 & 2,893 &, 382 \\
\hline Total & 36 & 119,56 & 6,20 & 18 & 119,22 & 6,42 & 18 & 119,89 & 6,14 &, 752 \\
\hline
\end{tabular}

En los 44 estudiantes evaluados de cuarto nivel básico, el puntaje promedio máximo se alcanzó en la habilidad motriz de locomoción; respecto a la diferencia más significativa que se puede apreciar entre géneros, es que las damas obtuvieron mejores resultados en las actividades relacionadas con la habilidad motriz de locomoción en comparación con los varones. Tabla 8.

Tabla 8

Valores medios, desviación estándar por genero del instrumento de desarrollo motor de los alumnos evaluados de cuarto básico.

\begin{tabular}{lcccccccccc}
\hline & \multicolumn{3}{c}{ Total } & \multicolumn{4}{c}{ Damas } & \multicolumn{7}{c}{ Varones } & p-valor \\
\cline { 2 - 13 } & $\mathrm{N}$ & $\times$ & $\mathrm{DE}$ & $\mathrm{N}$ & $\times$ & $\mathrm{DE}$ & $\mathrm{N}$ & $\times$ & $\mathrm{DE}$ & \\
\hline Locomoción & 44 & 46,16 & 2,46 & 22 & 46,45 & 1,87 & 22 & 45,86 & 2,949 &, 432 \\
\hline Manipulación & 44 & 30,48 & 2,06 & 22 & 30,73 & 1,96 & 22 & 30,23 & 2,181 &, 428 \\
\hline Equilibrio & 44 & 45,77 & 2,62 & 22 & 45,68 & 2,97 & 22 & 45,86 & 2,295 &, 821 \\
\hline Total & 44 & 122,41 & 5,35 & 22 & 122,86 & 5,75 & 22 & 121,95 & 4,999 &, 579 \\
\hline
\end{tabular}

\section{DISCUSIÓN}

El desarrollo motor ha sido objeto de estudio en diversas investigaciones del contexto académico, con el fin de conocer los niveles de desempeño relacionados con las habilidades motrices básicas que poseen los estudiantes, principalmente de primer ciclo básico, debido a que esta es la etapa más sensible para el desarrollo, en lo que respecta el proceso escolar (Durán, 1988). Gran parte de dichos estudios tienden a enfocarse en analizar los resultados por edad y género, con el fin de determinar una influencia significativa de dichas variables (Malina, 1986, Rosa, Rodríguez \& Márquez, 1996, Ruíz
\& Graupera, 2003, González, Cecchini, López \& Riaño, 2009).

En el presente estudio, se analizaron los datos obtenidos de una muestra de 190 estudiantes de primer ciclo básico, pertenecientes a tres establecimientos educacionales diferentes, de los cuales 95 son de sexo femenino y 95 de sexo masculino. Los resultados no arrojaron diferencias significativas entre ambos sexos, lo que se contrapone a investigaciones anteriores, como la de Ruíz y Graupera (2003), relacionada con competencia motriz y género, aplicada en la 
Díaz, S., Parada, B., Rojas, K., Frugone, D., Miño, M., \& Faúndez, C. (2019). Instrumento de evaluación para el desarrollo en edades tempranas de Frugone \& Miño. Revista Ciencias de la Actividad Física UCM, № 20(2), julio-diciembre, 1-10. DOI: http://doi.org/10.29035/rcaf.20.2.8

población escolar española, en la cual se encontraron grandes diferencias de disponibilidad motriz en diferentes tramos de edad y género. Además, en términos generales, las investigaciones manifiestan la presencia de una progresión lineal del desarrollo motor (González, Cecchini, López \& Riaño, 2009), es decir, una evolución directamente proporcional de acuerdo con la edad, situación similar a la que reportó el presente estudio, en donde los estudiantes de primero básico obtuvieron un promedio de puntuación de 117,0725, mientras que el de los de cuarto básico fue de 122,4091 puntos.

Otro dato importante se presentó en el estudio de González, Cecchini, López \& Riaño (2009), en el cual los resultados arrojaron que las mujeres manifiestan mejor desempeño que los varones, en lo que corresponde a habilidades motrices de locomoción; sin embargo, ocurre todo lo contrario en las acciones de manipulación. En el presente estudio se demuestra lo opuesto, ya que los varones obtuvieron mejores resultados que las mujeres en las pruebas de locomoción en prácticamente todos los niveles, mientras que las mujeres de tercero y cuarto básico alcanzaron un mejor desempeño en las de acciones de manipulación, con un promedio de puntuación de 30,44 y 30,74 puntos, contra un 30,06 y 30,23 puntos, respectivamente.

Considerando lo anterior, cabe destacar que numerosos estudios revisados han manifestado diferencias significativas del desarrollo motor en función de la edad, sexo y habilidad de los evaluados, acontecimiento que difiere a la presente investigación.

Por otra parte, la investigación se suma a lo manifestado por González, Cecchini, López \& Riaño (2009), los que destacan una progresión lineal del desarrollo motor, es decir, una evolución directamente proporcional de acuerdo a la edad; esto se ve reflejado en el puntaje promedio final $(117,07$ pts.) obtenido por los estudiantes de primero básico en contraste con los (122,40 pts.) logrados por alumnos de cuarto básico. Es aquí donde se hace referencia a las palabras de Doussoulin (2003): "Cada niño posee su propia secuencia de desarrollo, la cual está en directa relación con su maduración, por lo cual puede variar en características y calidad entre un niño u otro".

\section{CONCLUSIONES}

El test de Frugone \& Miño es un instrumento fiable para contribuir de forma segura y confiable en la evaluación de los niveles del desarrollo motor en estudiantes escolarizados de primer ciclo básico, lo que sugiere su uso y aplicación en el colegio para monitorizar el progreso del desempeño motor de los alumnos de ambos sexos.

Los resultados demuestran que no existen diferencias entre ambos géneros en relación a las habilidades motrices básicas de locomoción, manipulación y equilibrio, sin embargo, se observaron diferencias en los estudiantes de tercero básico, donde los varones obtuvieron mejores resultados en las actividades relacionadas a la habilidad motriz de locomoción en comparación con las damas; por otro lado, entre los estudiantes de segundo básico, las damas superan a los varones en la habilidad motriz de equilibrio, aunque estas diferencias no fueron estadísticamente significativas. 


\section{REFERENCIAS BIBLIOGRÁFICAS}

Aiken, L. (1996). Tests psicológicos de evaluación. México: Prentice-Hall.

Anastasi, A. (1986). Los tests psicológicos. Madrid: Aguilar.

Balestrini, M. (1997). Cómo se elabora el proyecto de investigación. (Para los estudios formulativos o exploratorios, descriptivos, diagnósticos, evaluativos, formulación de hipótesis causales, experimentales y los proyectos factibles). Caracas: BL Consultores Asociados. Recuperado de https:// issuu.com/sonia_duarte/docs/comose-elabora-el-proyecto-de-inve

Cronbach, LJ. (1984). Essentials of psychological testing. Nueva York: Gardner Press.

Doussoulin, S. (2003). Influencia del nivel socioeconómico y la estimulación ambiental en el desarrollo psicomotor en preescolares. Kinesiologia, (70), 15-17. Recuperado de https://pesquisa.bvsalud.org/portal/resource/pt/lil390334

Da Fonseca, V. (1996). Estudio y génesis de la Psicomotricidad. Barcelona: Inde.

Durán, M. (1988). El niño y el deporte. BarceIona: Paidós

Escriba, A. (1999). Psicomotricidad. Fundamentos teóricos aplicables en la práctica. Madrid: Gymnos.

Fritz, J. \& Vargas, R. (2013). Impacto de actividades lúdicas no competitivas sobre las habilidades motoras gruesas en niños y niñas pre escolares. Revista Ciencias de la Actividad Física UCM, 14(1) 31-37. Recuperado de http://repositorio.ucm.cl/bitstream/ handle/ucm/1381/vargas_r_impacto. pdf? sequence $=1$ \&isAllowed $=y$
Fuentes-Lopez, J., Puño, L., De Arruda, M., Cossio-Bolaños, M. (2014). Reproductibilidad de un cuestionario que valora la actividad física en adolescentes escolares. Revista Peruana de Ciencias de la Actividad Física y del Deporte 7(4), 115-120. Recuperado de https://www.researchgate.net/ publication/280113187_Reproductibilidad_de_un_cuestionario_que_valora_la_actividad_fisica_en_adolescentes_escolares

Gallahue, D.L., Y Ozmun, J.C. (2006). Understanding motor development: Infants, children, adolescents and adults (6a ed.). Boston: McGraw Hill.

González, C., Cecchini, J., López, J. \& Riaño, C. (2009). Disponibilidad de habilidades motrices en escolares de 4 a 14 años. Aplicabilidad del Test de desarrollo motor grueso de Ulrich. Aula Abierta, 37(2), 19-28.

Le Boulch, J. (1995). El desarrollo psicomotor desde el nacimiento hasta los 6 años. Consecuencias educativas. Barcelona: Paidotribo.

Le Boulch, J. (1997). El movimiento en el desarrollo de la persona. Barcelona: Paidotribo

Ledesma, R., Molina, G.\&Valero, P. (2002). Análisis de consistencia interna mediante Alfa de Cronbach: un programa basado en gráficos dinámicos. Psico-USF, 7(2), 143-152. https://dx.doi.org/10.1590/ S1413-82712002000200003

Malina, R.M (1986). Genetics of motor develop ment and perfomance. En R.M. Malina, \& C. Bouchard, C., Sport and human genetics (4a Ed.) (pp. 23-58.). Champaign IL: Human Kinetics. 
Pascucci, MC., Lejarraga, H., Kelmansky, D, Álvarez, M, Boullón, M, Breiter, P, ... Waisburg, H. (2004). Validación de la prueba nacional de pesquisa de trastornos de desarrollo psicomotor en niños menores de 6 años. Revista chilena de pediatría, 75(1), 75-76. https://dx.doi.org/10.4067/s0370 41062004000100012

Rosa, J., Rodríguez, L. P., Márquez, S. (1996). Evaluación de la ejecución motora en la edad escolar mediante los test motoros de Lincoln-Oseretsky 129. European Journal of Human Movement, 2, 129-147. Recuperado de https://recyt.fecyt.es/index.php/ejhm/article/ view/56120/33932
Ruíz, L.M. \& Graupera, J.L. (2003). Competencia motriz y género entre escolares españoles. Revista Internacional de Medicina y Ciencias de la Actividad Física y el Deporte, 3(10), 101-111. Recuperado de http://cdeporte.rediris. es/revista/revista10/artcompetencia. html

Ulrich, D. (2000). Test of Gross Motor Development. Examiner's Manual. Austin: Pro-ed.

\section{Dirección para correspondencia}

Cesar Faúndez Casanova

Magíster en Ciencias de la Actividad Física

Depto. Ciencias de la Actividad Física

Facultad de Ciencias de la Educación

Universidad Católica del Maule

Talca, Chile

ORCID iD: https://orcid.org/0000-0003-4501-4169

https://www.researchgate.net/profile/Cesar_Faun-

dez_Casanova2

Contacto:

cfaundez@ucm.cl

Recibido: 04-08-2019

Aceptado: 04-12-2019 